\title{
PRIME Regimen
}

National Cancer Institute

\section{Source}

National Cancer Institute. PRIME Regimen. NCI Thesaurus. Code C9631.

A chemotherapy regimen consisting of procarbazine, ifosfamide, and methotrexate that may be used in the treatment of bronchogenic carcinoma. 